\title{
Metodología de costos para los productos agrícolas, basada en las normas internacionales de contabilidad. Una aplicación en las ciruelas europeas variedad D’Agen*
}

Cost methodology for agricultural products, based on international accounting standards. An application in European plums kind D'Agen

Sandra Alvear Vega a

Universidad de Talca, Chile

salvear@utalca.cl

ORCID: http://orcid.org/0000-0002-2249-0720

Katerin Figueroa Salinas

Universidad de Talca, Chile

ORCID: http://orcid.org/0000-0002-6591-1409
DOI: https://doi.org/10.11144/Javeriana.cc19-48.mcpa Redalyc: http://www.redalyc.org/articulo.oa? id $=151557795004$

Fecha de recepción: 10 Diciembre 2017 Fecha de aprobación: 05 Septiembre 2018 Fecha de publicación: 20 Diciembre 2018

\section{Resumen:}

Este artículo expone la aplicación de una metodología para medir el costo de producción del kilo de fruta deshidratada, aplicando las Normas Internacionales de Contabilidad, en una plantación de Ciruelo D’Agen, en Chile. Se muestra el costo por cada árbol, ciruelo, hasta el inicio de la primera cosecha, clasificado en fijo y variable. Además, se determina el impacto de la depreciación del árbol, en el kilo de ciruela deshidratada. Finalmente, se determinó el costo del kilo de ciruela deshidratada. El costo total del kilo de ciruela deshidratada es intensivo en costos fijos, lo anterior, tiene relevancia en la gestión de la fruticultura en Chile, pues una disminución en los niveles de cosecha o de precios afecta a los agricultores en la viabilidad de la inversión en el mediano y largo plazo, de ahí la importancia de disponer de una metodología de costos, que incorpore la dimensión técnica, económica y financiera. Palabras clave: contabilidad, gestión de la producción, agricultura.

\section{Abstract:}

This article exposes the application of a methodology to measure the cost of production of the kilo of dehydrated fruit, applying the International Accounting Standards, in a plantation of Ciruelo D'Agen, in Chile. The cost is shown for each tree, plum tree, until the beginning of the first harvest, classified as fixed and variable. In addition, the impact of the depreciation of the tree is determined in the dehydrated plum kilo. Finally, the cost of the dehydrated plum kilo was determined. The total cost of the kilo dehydrated plum is intensive in fixed costs, the previous thing, has relevance in the management of the fruit growing in Chile, because a decrease in the levels of harvest or of prices affects the farmers in the viability of the investment in the medium and long term, hence the importance of having a cost methodology that incorporates the technical, economic and financial dimension.

Keywords: accounting, production management, farming.

\section{Introducción}

La fruticultura en Chile es una actividad que enfrenta importantes desafíos, entre otros, la estacionalidad del empleo, que de proyectarse en el futuro irían deteriorando la rentabilidad del sector y una desaceleración del crecimiento del sector (Anriquez et ál., 2016). Por ello, disponer de información relevante sobre los costos de producción es un factor relevante para propiciar en el mediano y largo plazo, la búsqueda del crecimiento y la competitividad, de pequeños y medianos productores agrícolas, a través de la toma de decisiones.

En la producción de ciruelas deshidratadas es necesario valorizar dos activos: el primero de ellos, un activo no corriente, capitalizable, el Ciruelo, clasificado como activo biológico según la norma contable. El segundo,

Notas de autor

\footnotetext{
a Autor de correspondencia. Correo electrónico: salvear@utalca.cl
} 
un activo corriente inventariable, la Ciruela Deshidratada. En tal sentido, es necesario integrar desde un enfoque multidisciplinario los avances alcanzados desde el punto de vista contable y agrícola.

Una mirada sistémica en el sector agrícola muestra que la contabilidad añade información significativa para explicar y predecir la viabilidad de las explotaciones agrícolas, y por ello es útil para éstas y para los demás agentes que intervienen en el sector, tales como bancos, inversionistas, proveedores, y en general para la toma de decisiones sobre la base de una información sólida (Argilés-Bosch, 2007). En la misma línea, existen estudios que muestran que la Contabilidad, como sistema de información, contribuye a disminuir la presión de los acreedores, dado que permite a los directivos comunicar la información financiera, necesaria para evaluar el desempeño de la empresa y su salud financiera, y a futuro las empresas pueden acceder a mejores condiciones de endeudamiento y un menor costo de la deuda (García et ál., 2018). Esta situación es particularmente importante en el sector agrícola, caracterizado por su intensidad en financiamiento externo. Muchas empresas agrícolas, generalmente de naturaleza familiar, están respaldas por préstamos y en ellas la reinversión se realiza con endeudamiento externo. A nivel interno, la contabilidad de costo tiene mayor relevancia, y es aplicada por las empresas agrícolas para efectos de controlar los márgenes de venta y flujos de caja (Jack et ál., 2018).

Desde el punto de vista de las Normas Internacionales de Contabilidad -nic- las plantas para producir frutos se definen como aquellas utilizadas en la producción o suministro de productos agrícolas, de la que se espera producir frutos durante más de un periodo, y que no está destinada a la venta como una planta viva o cosechada como un producto agrícola, a la excepción de las venta de desperdicio imprevisto.

Las plantas para producir frutos, hasta el 31 de diciembre de 2015, se valoraban de acuerdo con la NIC 41: Activos Biológicos.

De acuerdo con la Norma Internacional de Contabilidad 41, un activo biológico se medirá tanto en el reconocimiento inicial como al final del periodo, a su valor razonable menos los costos de ventas. Lo anterior, siempre y cuando, se pueda medir el precio del activo biológico en análisis en un mercado activo; en caso contrario, es decir, que no exista la posibilidad de contar con los precios de un mercado activo, la norma plantea una excepción a la utilización del valor razonable, admitiendo el valor costo menos la depreciación acumulada y menos cualquier pérdida por deterioro que presente el activo.

El método del valor razonable es el más recomendable para efectos de valoración de los activos biológicos, dado el impacto que produce en el valor del patrimonio de las empresas agrícolas, sin embargo, aun existiendo un mercado activo, las empresas agrícolas mayoritariamente optaban por el método del costo (Arimany et ál. 2013). En la misma línea, existe evidencia que los activos biológicos influencian negativamente la capacidad de predicción cuando están valorados a costo histórico y, la aplicación del valor razonable vuelve positiva dicha influencia negativa (Argilés-Bosch et ál., 2017).

Sin embargo, a partir de enero de 2017, las plantas para producir frutos se valorizan de acuerdo con la NIC 16: Propiedad Planta y Equipos. En tanto, el Ciruelo desde el punto de vista contable es clasificado como un Activo no Corriente y por ello, todos los desembolsos incurridos hasta la primera producción se capitalizan como Activo Fijo (Activo no Corriente), se deprecian, dado que prometen beneficios futuros por más de un periodo, y se reconoce la pérdida por deterioro de valor. La depreciación se inicia con la primera producción y su importe es inventariado como costo indirecto en la valorización del kilo de Ciruela Deshidratada.

En la valorización de los productos agrarios, a partir de su cosecha se aplica la NIC 2: Inventarios. El kilo de Ciruela Deshidratada desde el punto de vista contable es clasificado como un Activo Corriente (NIC 2), es decir, a partir de la primera producción del Ciruelo son costos inventariables o costos del producto todos los desembolsos e importes hasta que el producto quede disponible para la venta, incluida el mantenimiento de los Ciruelos, su depreciación, la mano de obra de cosecha, entre otros. Al momento de la venta del producto (kilo de Ciruela Deshidratada), el costo de producción (activo) se clasificará como costos de venta (gasto) en el Estado de Resultado. Según la NIC 2 el costo de producción de un producto comprenderá todos los 
desembolsos o importes derivados de su adquisición y transformación, así como otros desembolsos o importes en los que se haya incurrido para darles su condición y ubicación actual.

La ciruela deshidratada es un alimento cuyo consumo ha crecido en los últimos años, dado sus múltiples beneficios para la salud. Lo anterior se ve reflejado en el aumento del volumen de las exportaciones mundiales, un 72,2\% entre los años 2001 y 2010 (Tapia \& González, 2012). Chile presenta condiciones favorables para el desarrollo económico de la fruticultura, gracias a las ventajas comparativas, tales como las buenas condiciones de suelo, clima y fitosanitarias. Adicionalmente, la posibilidad de producción en contraestación respecto del hemisferio norte y la amplia red de acuerdos comerciales, entre otras. A nivel país, destaca la Región del Maule, dada su especialización productiva, con el $41 \%$ del total de la superficie de la explotación dedicado al rubro frutícola y el $70 \%$ de su producción es destinada al mercado de exportación. Sin embargo, es necesario impulsar la productividad en los productores concentrados en rangos de superficie de explotación menores a 50 hectáreas (Riveros et ál., 2016).

Una de las plantaciones frutícola que se ha intensificado en el país es el Ciruelo, específicamente, el perteneciente a la familia de las Rosáceras, variedad D’Agen, cuya producción es consumida nacional e internacionalmente como fruta deshidratada. Es así como en Chile existen 12.384 hectáreas plantadas, de ellas $97,6 \%$ se concentra en la zona centro sur de Chile y al año 2010 representaba el 0,7\% de la superficie mundial plantada (Tapia \& González, 2012).

La ciruela europea, variedad D'Agen, se conoce con el nombre científico Prunus Domestica, es de originaria del oeste de Asia, con propagación a Europa. Su propagación es vegetativa y por semilla, con dos flores en cada yema. Su fruto es de color azul violeta, con un hueso en su interior. Se adapta a diferentes tipos de suelo, excepto los suelos ligeros, áridos y muy compactos. Es resistente al frío invernal, soporta la carencia de agua y es tolerante a la humedad. Es especial para climas mediterráneos, con 325 a 600 milímetros de lluvia por año (Cobianchi et ál., 1989).

La ciruela deshidratada, variedad d'Agen, es valorizada es varios países para su consumo por las múltiples propiedades energéticas que posee, factor determinante en el aumento de las exportaciones (Tapia \& González, 2012). Sin embargo, el sector frutícola en general enfrenta importantes desafíos, desde el punto de vista de la eficiencia productiva - económica, social y ambiental. Por ejemplo, el uso de agroquímicos y maquinaria para el preparado del suelo y mantención de los ciruelos, entre otros, deben ser evaluados cuidadosamente para evitar excesivos consumos que afectan la rentabilidad de esta actividad (Marvinney et ál., 2015). Los riesgos asociados a dichos desafíos disminuirán si los pequeños y medianos productores agrícolas cuentan con información para planificar, presupuestar, gestionar y controlar todas las actividades, sean estás productivas o administrativas, promoviendo de esta forma la productividad como impulsor del crecimiento y de competitividad (Mallo et ál., 2000).

El presente artículo define una metodología de costos, que incorpora las especificidades de los procesos agrícolas y la evolución de la contabilidad de costos. Así se hace un aporte al campo de la generación de información para medir la eficiencia y productividad que enfrentan los pequeños y medianos productores frutícolas.

Además de la presente introducción este artículo se estructura así: en la primera sección se hace la descripción metodológica del estudio de costos, identificando: caracterización del objeto de costos, nivel de actividad, sistema de costeo y valorización. En la segunda sección se presentan los resultados y la discusión, para terminar con la exposición de las conclusiones.

\section{Metodología}

Se trata de un estudio de costos, cuyos datos se recolectaron entre el 01 de agosto y 31 de octubre de 2016. 
Caracterización del objeto de costo. El objeto de costos del presente trabajo corresponde a la explotación de 9 hectáreas de plantación de Ciruelos Europeos, variedad D’Agen, ubicada en la séptima región, comuna de Maule, en la localidad de Santa Rosa de Lavaderos, Chile. Esta plantación se encuentra localizada en una llanura cerca al río Maule, requiriendo poco riego, al ser tierras fértiles, situación que es favorable para el cultivo de la variedad de ciruela D’Agen, el cual no soporta suelos ligeros, áridos y compactos (Cobianchi et ál., 1989).

Nivel de actividad del objeto de costo. La unidad de costos es la hectárea de Ciruelos Europeos, variedad D’Agen. Se trabajó sobre la base de un estándar teórico de eficiencia, por hectárea, equivalente a 515 plantas, con un $97 \%$ de plantas producidas al finalizar el segundo año y un $3 \%$ de pérdida normal. Lo anterior, según la información proporcionada por la empresa en estudio. Después de un periodo de tres años de desarrollo se obtiene la primera cosecha, con una vida útil productiva de 16 años.

Sistema de costeo y valorización. Se empleó el método de costeo absorbente, caracterizado por incluir los costos directos e indirectos fijos y variables (Alvear \& Rodríguez, 2006). Los costos indirectos fijos y variables se aplicaron, a partir de una tasa definida como la fracción entre los desembolsos e importes (depreciación) totales y el volumen esperado del causante del costo, en este caso las 9 hectáreas plantadas.

Para su valorización se trabajó sobre la base de costos estándares, es decir, los rendimientos que los administradores pueden alcanzar a través de esfuerzos realistas (Horngren et ál., 2007). El estándar de precio de la mano de obra y los estándares de eficiencia de cada proceso (número de plantas por hectárea, cantidad de insumos empleados, número de horas hombre y máquina) se obtuvieron del sistema de información de la empresa en estudio. Los estándares de precio de los insumos se obtuvieron del mercado, a través de cotizaciones.

A partir del 01 de agosto de 2016 la recolección de los datos se efectuó sistemática y cronológicamente por un profesional externo, bajo la supervisión externa de un experto, utilizando las técnicas de análisis documental, observación directa no participativa y el análisis de los procesos. Internamente, para definir los procesos productivos se contó con la colaboración de un Ingeniero Agrónomo y un Técnico Agrícola, y se entrevistó a todo el personal que participa directamente en ellos.

\section{Resultados y discusión}

En primer lugar es importante destacar que el sector silvoagropecuario (agroalimentario y forestal) es relevante en la actividad económica chilena, aportando para el periodo 2015 el 2,4\% del Producto Interno Bruto (PIB) nacional, considerando sólo la producción primaria. Para el mismo año la variación anual del PIB silvoagropecuario ascendió a 5,6\%, superior a la variación del 2,1\% anual del PIB nacional para el mismo año. La zona centro sur de Chile (Región: Metropolitana, O’Higgins, Maule y Biobío) concentra el 60,8\% de la producción del sector a nivel país, y los pequeños y medianos empresarios agrícolas tiene un rol fundamental, al ser un sector que está siendo impulsado por la competitividad nacional e internacional (Gumucio, 2017).

Los costos directos se pueden relacionar con el nivel de actividad - la hectárea de ciruelos plantada - y son equivalentes a: los insumos empleados en la preparación, plantación y mantención de los ciruelos (plantas, fungicidas, fertilizantes, pesticidas y herbicidas). El costo total de insumos variables por hectárea plantada, acumulados hasta la finalización del segundo año, asciende a US\$3.632,22, incluido el costo de transporte. La planta de Ciruelo tiene un costo de US\$ 4,15 la unidad (US\$ 3,8 + US\$ 0,3541 de transporte) y US \$2.077,05 por hectárea plantada, los fungicidas ascienden a US\$152,64, los fertilizantes US\$ 404,61, los pesticidas US\$456,92, los herbicidas US\$15, filtro regulador US\$252,83 y acaricida 273,19. Ver la tabla 1. 
TABLA 1

Estructura de costo de insumos de la hectárea de Ciruelos*

\begin{tabular}{|l|r|}
\hline Plantas (US \$ / Unidad) & $2.077,05$ \\
\hline Fungicida: Captan 80 (Kg /hectárea) & 66,00 \\
\hline Bumper (Lts / hectárea) & 36,66 \\
\hline Acoidal WG (Kg/ hectárea) & 102,13 \\
\hline Fertilizante: Trainer (Lts /hectárea) & 302,48 \\
\hline Wuxal Zinc (Lts / hectárea) & 456,92 \\
\hline Pesticida: Cobre nordox (Kg /hectárea) & 15,00 \\
\hline Herbicida: Roundup Ultramax (Kg /hectárea) & 252,83 \\
\hline Filtro regulador: Auxym & 273,19 \\
\hline Acaricida: Envidor 240 SC & $3.632,22$ \\
\hline Total insumos & \\
\hline
\end{tabular}

Fuente: elaboración propia, a partir de los datos del estudio. Las marcas de los productos son referenciales, no se están recomendando. ${ }^{*}$ Costos insumos: preparación, plantación y mantención del Ciruelo.

También, constituye costo directo la mano de obra empleada en el proceso de preparación, plantación y mantención de los ciruelos (rotura, subsolado, rastraje, trazado, estancado, hoyadura, plantación, acequiadura, riego, aplicación de agroquímicos y aplicación de fertilizantes). Los costos de preparación de suelo ascienden a US\$12,87, de la plantación US\$ 53,82 y de la mantención de los Ciruelos asciende a US $\$ 572,94$, todos los valores son fijos por hectárea plantada, acumulado hasta la finalización del segundo año. Ver la tabla 2. 
TABLA 2

Estructura de costo de mano de obra directa de la hectárea de ciruelos*

\begin{tabular}{|c|c|}
\hline Preparación de Suelo & \\
\hline Rotulado (US \$ $497 / 180$ Hora Hombre)* 2 horas & 5,52 \\
\hline Subsolado (US \$ $497 / 180$ Hora Hombre) $* 1,777$ horas & 4,9 \\
\hline Rastraje (US \$ $497 / 180$ Hora Hombre)* 0,888 horas & 2,45 \\
\hline Sub Total 1: Costo mano de obra preparación de suelo & 12,87 \\
\hline Proceso de Plantación & \\
\hline Trazado y estancado (US $\$ 497 / 180$ Hora Hombre) $* 3$ horas & 8,28 \\
\hline Hoyadura (US $\$ 497 / 180$ Hora Hombre) ${ }^{*} 8$ horas & 22,08 \\
\hline Plantación (US \$ $497 / 180$ Hora Hombre)* 8 horas & 22,08 \\
\hline Acequiadura (US \$ $497 / 180$ Hora Hombre) $* 0,5$ horas & 1,38 \\
\hline Sub- total 2: Costo mano de obra plantación & 53,82 \\
\hline Mantención de Ciruelos & \\
\hline Primer riego (US \$ 497/180 Hora Hombre) * 16 horas, año 1 & 44,18 \\
\hline Riegos mantención (US \$ 497/180 Hora Hombre)* 142,5 horas, año 1 y 2 & 393,46 \\
\hline Aplicación agroquímicos (US $\$ 497 / 180$ Hora Hombre) $* 17$ horas, año 1 y 2 & 46,94 \\
\hline Aplicación fertilizantes (US $\$ 497 / 180$ Hora Hombre)* 32 horas, año 1 y 2 & 88,36 \\
\hline Sub -total 3 : mano de obra directa mantención ciruelos & 572,94 \\
\hline Costo total de Mano de Obra Directa (St 1 + St2 + St3) & 639,63 \\
\hline
\end{tabular}

Fuente: elaboración propia, a partir de los datos del estudio.

* Costo de mano de obra directa, proceso preparación, plantación y mantención.

Los costos indirectos variables son aquellos que no se pueden identificar directamente con el nivel de actividad -la hectárea de ciruelos plantada - y varían en relación con dicho nivel de actividad. Específicamente, para el caso en estudio el costo asociado a la lienza de alambre y estanquillas de coligue ascendente a US\$ 7,96. Ver la tabla 3. 
TABLA 3

Estructura de Costos Indirectos de Producción (CIP) de la hectárea de Ciruelos*

\begin{tabular}{|l|c|}
\hline Lienza de alambre (US \$ 5,19/9 hectárea) & 0,58 \\
\hline Estaquilla de coligüe (US \$ 66,39/9 hectárea) & 7,38 \\
\hline CIP Variable & 7,96 \\
\hline
\end{tabular}

\footnotetext{
Fuente: elaboración propia, a partir de los datos del estudio.

* Costos Indirectos de Producción (CIP): preparación, plantación y mantención del ciruelo.
}

Los costos indirectos fijos son aquellos que no se pueden identificar directamente con el nivel de actividad -la hectárea de ciruelos plantada- y se mantienen constantes para un rango de relevancia dado. El costo de mano de obra indirecta asciende a US\$176,91, el costo indirecto asociado a la preparación de suelo US\$ 14.793,93, incluye el costo alternativo del terreno, la depreciación de herramientas y arriendo del Bulldog. Es importante destacar que los terrenos no se deprecian, en tanto, el costo alternativo del terreno sólo debe ser considerado para efectos de la evaluación económica, y no para efectos de valorización de los inventarios, en la presentación de los Estados Financieros. Desde el punto de vista económico, el agricultor renuncia a una segunda opción, el arriendo, al elegir la plantación. El costo indirecto fijo asociado al proceso de plantación asciende a US\$ 0,1. El costo indirecto fijo asociado proceso de mantención asciende a US\$4,89. Ver la tabla 4.

TABLA 4

Estructura de Costos Indirectos de Producción (CIP) de la hectárea de Ciruelos*

\begin{tabular}{|c|c|}
\hline Mano de Obra Indirecta & \\
\hline Supervisión: Ingeniero Agrónomo (US\$ $265,4 \times 3$ visita $\times 2$ años) $/ 9$ hectáreas & 176,91 \\
\hline Sub- total 1: CIP Fijo & 176,91 \\
\hline \multicolumn{2}{|l|}{ Preparación de Suelo } \\
\hline Costo altemativo del terreno (US\$ 589,73 por hectárea x 24 meses) & $14.153,52$ \\
\hline Depreciación Arado (US\$ $3.980,66 / 10.800$ horas) x 2 horas por hectárea & 0,74 \\
\hline $\begin{array}{l}\text { Depreciación Arado Escarificador (US\$ } 3.538,36 / 10.800 \text { horas) x 1,78 horas por } \\
\text { hectárea }\end{array}$ & 0,58 \\
\hline Depreciación Rastra (US\$ $6.418,05 / 10.800$ horas) x 0,88 horas por hectárea & 0,53 \\
\hline Arriendo Bulldog (US\$ 5.747,07/9 hectárea) & 638,56 \\
\hline Sub- total 2: CIP Fijo & $14.793,93$ \\
\hline \multicolumn{2}{|l|}{ Proceso de Plantación } \\
\hline Depreciación Pala (US\$ 14,98 x 6 unidades/ 12.960 horas) x 8 horas por hectárea & 0,06 \\
\hline Depreciación Arado Tatu (US\$ $884,59 / 10.800$ horas) x 0,5 horas por hectárea & 0,04 \\
\hline Sub- total 3: CIP Fijo & 0,1 \\
\hline \multicolumn{2}{|l|}{ Mantención de ciruelos } \\
\hline Depreciación Pala de Riego (US\$ $14,48 / 12.960$ horas) x 158,5 horas por hectárea & 0,18 \\
\hline Depreciación Pulverizadora (US\$ $86,71 / 12.960$ horas) x 17 horas por hectárea & 0,11 \\
\hline Depreciación Tractor Massey (US $\$ 27.165,88 / 32.400$ horas) x 5,5 horas por hectárea & 4,6 \\
\hline CIP Fijos & 4,89 \\
\hline
\end{tabular}

Fuente: elaboración propia, a partir de los datos del estudio.

* Costos Indirectos de Producción (CIP): preparación, plantación y mantención del ciruelo. 
Finalmente, el costo total por hectárea plantada asciende a US\$19.255,64 y US\$ 38,51 por cada planta de Ciruelo D’Agen al finalizar el segundo año de crecimiento. Dicho costos se constituyen de la siguiente forma: el costo directo de insumos asciende a US\$3.632,22, el costo de mano de obra directa US\$ 639,63 y los costos indirectos US\$14.983,79, por hectárea plantada. Del total de costos por hectárea plantada el $81 \%$ son fijos y el $19 \%$ son variables. Ver la tabla 5 .

TABLA 5

Ficha técnica y estructura de costo de la hectárea de Ciruelos

\begin{tabular}{|l|r|r|r|}
\hline \multicolumn{2}{|l|}{ Resumen estructura de costos de una hectárea de ciruelos } \\
\hline Árbol : Frutícola & Tipo: Ciruelo Europeo & Variedad: D'Agen & Moneda: US\$ $=\$ 678,28$ \\
\hline Costos total de Insumos Directos & $3.632,22$ \\
\hline Costo total de Mano de Obra Directa & 639,63 \\
\hline Costo total de Costos Indirectos & $14.983,79$ \\
\hline \multicolumn{4}{|r|}{ Costo total por hectárea plantada, finalizado el año 2 } \\
\hline Costo unitario de una planta de Ciruelo D'Agen al finalizar el año 2 & $19.255,64$ \\
\hline
\end{tabular}

Fuente: elaboración propia, a partir de los datos del estudio.

Los resultados de un estudio realizado por la Oficina de Estudios y Política Agrarias (ODEPA) en 2015 sobre el costo de la Ciruela variedad D’Agen, para secado en la Región de O’Higgins, muestra que los costos totales por hectárea asciende a US\$3.782,56. Valor actualizado al año 2016, según variación acumulada del Índice de Precio al Consumidor de un 15\%, calculada por el Instituto Nacional de Estadística. Con un rendimiento de $9.000 \mathrm{~kg} /$ ha para la producción del primer año. Sin embargo, dicho estudio no distingue el costo del producto Ciruela para Secado (inventario), de la inversión asociada al Ciruelo (Activo Fijo), hasta su edad de producción, es decir, al finalizar el segundo año de crecimiento.

Desde el punto de vista financiero y económico, es importante destacar que el Ciruelo es un Activo Fijo, es decir, un recurso controlado por la entidad como resultado de sucesos pasados, del que la entidad espera obtener, a futuro, beneficios económicos. Para el caso de los Ciruelos D’Agen, dichos beneficios económicos llegan a la empresa usándolos en la producción de ciruelas, a vender por dicha entidad. Por ello, se deben depreciar a partir del momento que estén apto para producir, en función del periodo máximo productivo, es decir, 16 años. Para el caso en estudio la depreciación anual de cada planta de Ciruelo asciende a US\$2,41 (38,51 / 16 años). La depreciación del Ciruelo pasa a ser un costo indirecto fijo del kilo de ciruela y se entiende como la distribución sistemática del importe de un activo a lo largo de su vida útil (NIC 16).

Para efectos contables todos los desembolsos e importes (depreciación) incurridos a partir del primer periodo de producción, pasan a ser costos del kilo de ciruela deshidratada (NIC 16, NIC 2).

Por ello, desde el punto de vista de la valoración del costo del kilo de ciruela deshidratada la depreciación del árbol ciruelo es un costo indirecto de producción (CIP), equivalente a US\$ 1.203,48 por hectárea. Adicionalmente, forman parte del CIP la depreciación de máquinas, herramientas y mano de obra indirecta, asociadas a los procesos de cuidado, mantención, cosecha y secado. Ver la tabla 6A. 
TABLA 6A

Estructura de Costos Indirectos de Producción*

\begin{tabular}{|c|c|}
\hline CIP Fijo. Depreciación Ciruelos & \\
\hline $\begin{array}{l}\text { Depreciación Ciruelos (US\$19.255,64 costo total por hectárea plantada/ } 16 \text { años de } \\
\text { vida útil) }\end{array}$ & $1.203,48$ \\
\hline Sub- total 1: CIP Fijo & $1.203,48$ \\
\hline Proceso de Cuidados - Depreciación & \\
\hline $\begin{array}{l}\text { Depreciación Tijerones de poda (US } \$ 26,62 / 8.640 \text { horas) } * 40 \text { horas por hectárea)*5 } \\
\text { Tijerones }\end{array}$ & 0,62 \\
\hline Depreciación Brocha de 3 pulgadas (US $\$ 6,80 / 2.160$ horas) $* 40$ horas por hectárea & 0,13 \\
\hline Depreciación Chipiadora(US $\$ 8.403,61 / 21.600$ horas) $* 4,4$ horas por hectárea & 1,71 \\
\hline $\begin{array}{l}\text { Depreciación Arado Escarificador(US\$ } 3.538,36 / 10.784 \text { horas) } * 1,8 \text { horas por } \\
\text { hectárea }\end{array}$ & 0,59 \\
\hline Depreciación Rastra (US\$ 6.418,05/ 10.792 horas) * 0,9 horas por hectárea & 0,54 \\
\hline Proceso de Cuidados-Mano de Obra & \\
\hline Poda y pintado(US $\$ 497 / 180$ Hora Hombre)* 40 horas & 110,44 \\
\hline Chipiar (US \$ $497 / 180$ Hora Hombre) ${ }^{*} 4,4$ horas & 12,15 \\
\hline Subsolar (US \$ 497/ 180 Hora Hombre)* 1,8 horas & 4,97 \\
\hline Rastraje (US \$ 497/180 Hora Hombre)* 0,9 horas & 2,49 \\
\hline Proceso de Cuidados - Insumos CIP Variable & \\
\hline Agroquímicos (US $\$ 7.801,42 / 9$ hectáreas) & 866,82 \\
\hline Proceso de Mantenciones - Depreciación & \\
\hline Depreciación Turbo (US\$ 6.929,29/21.600 horas) * 13,2 horas por hectárea & 4,23 \\
\hline Depreciación Pala de Riego (US $\$ 14,48 / 11.534$ horas) $* 75,5$ horas por hectárea & 0,09 \\
\hline Sub- total 2: CIP Fijo - Variables & $1.004,78$ \\
\hline
\end{tabular}

Fuente: elaboración propia, a partir de los datos del estudio.

* Costos Indirectos de Producción (CIP) del kilo de Ciruela Deshidratada: proceso: cuidado, mantención y cosecha.

También, forman parte del CIP fijo, la malla raschel, plástico blanco, depreciación del tractor, sueldo del profesional agrónomo y del encargado de producción. Totalizando, US\$ 3.845,38 por hectárea, de ellos US $\$ 4.020,28$ son variables y US\$2.543,35 son fijos. Ver la tabla 6B. 
TABLA 6B

Estructura de Costos Indirectos de Producción*

\begin{tabular}{|c|c|}
\hline Proceso de Mantenciones - Mano de Obra & \\
\hline Primer riego (US \$ $497 / 180$ Hora Hombre) x 8 horas & 22,09 \\
\hline Riego de mantención (US \$ 497/180 Hora Hombre) x 67,5 horas & 186,38 \\
\hline Aplicación agroquímicos (US $\$ 497 / 180$ Hora Hombre) x 13,2 horas & 36,45 \\
\hline Aplicación abono (US \$ 497/180 Hora Hombre) x 16 horas & 44,18 \\
\hline Sub- total 3: CIP Fijos & 293,42 \\
\hline Proceso de Secado y Recolección - Depreciación & \\
\hline $\begin{array}{l}\text { Depreciación Rastrillo (US\$ } 13,62 / 4.320 \text { horas) x } 2,7 \text { horas por hectárea) x } 4 \\
\text { rastrillos) }\end{array}$ & 0,03 \\
\hline Arriendo Tractor (US \$ 442,30/9 hectáreas) & 49,14 \\
\hline Arriendo Colosos (US \$ 530,75/9 hectáreas) & 58,97 \\
\hline Arriendo Maquina Recolectora (US\$ $6.796,94 / 9$ hectáreas) & 755,22 \\
\hline Proceso de Secado - CIP Variables & \\
\hline Malla Raschel (US\$ $19.460,99 / 9$ hectáreas) & $2.162,33$ \\
\hline Plástico Blanco (US\$1.149,97/9 hectáreas) & 127,77 \\
\hline Sub- total 4: CIP fijos - variables & $3.153,46$ \\
\hline Otros CIP que participan en varios procesos & \\
\hline Depreciación Tractor (US $\$ 27.165,88 / 32.351$ horas) x 22,2 horas por hectárea & 18,64 \\
\hline Sueldo agrónomo (US\$ 796,13 costo al año por las visitas / 9 hectáreas) & 88,46 \\
\hline Sueldo encargado de Producción (US\$ 7.251,36 costo al año/ 9 hectáreas) & 805,71 \\
\hline Sub total 5: CIP Fijos & 912,81 \\
\hline Total CIP (St 1 + St 2+ St 3 + St 4 + St 5) & $6.563,63$ \\
\hline Total CIP Variables & $4.020,28$ \\
\hline Total CIP Fijos & $2.543,35$ \\
\hline
\end{tabular}

Fuente: elaboración propia, a partir de los datos del estudio.

${ }^{*}$ Costos Indirectos de Producción del kilo de Ciruela Deshidratada. Proceso: Cuidado, Mantención y Cosecha.

El costo de mano de obra directa asociada a los procesos de cosecha y secado asciende a US\$1.066,15 y US\$217,65 por hectárea respectivamente. El costo total de Mano de Obra Directa asciende a US\$1.283,8 por hectárea. Ver la tabla 7. 
TABLA 7

Estructura de costos de Mano de Obra Directa del kilo de Ciruela Deshidratada*

\begin{tabular}{|c|c|}
\hline Proceso de Cosecha & \\
\hline $\begin{array}{l}\text { Mano de obra Variable ( } 25 \text { personas * US } \$ 1,47 \text { por caja * } 22,5 \\
\text { cajas en promedio por persona * } 10 \text { dias de cosecha)/ } 9 \text { hectáreas) }\end{array}$ & 918,75 \\
\hline $\begin{array}{l}\text { Mano de Obra Fija (6 personas * US } \$ 22,11 \text { diario * } 10 \text { días de } \\
\text { cosecha) } / 9 \text { hectáreas) }\end{array}$ & 147,4 \\
\hline Sub- total 1: Costo mano de obra cosecha & $1.066,15$ \\
\hline Proceso de Secado & \\
\hline $\begin{array}{l}\text { Mano de Obra secado ( } 6 \text { personas para el secado * US } \$ 17,69 \\
\text { diario * } 18 \text { días de secado en promedio) } / 9 \text { hectáreas) }\end{array}$ & 212,28 \\
\hline $\begin{array}{l}\text { Traslado de frutas en el secado (US } \$ 497 / 180 \text { Hora Hombre) } \\
* 17,5 \text { horas) } / 9 \text { hectáreas }\end{array}$ & 5,37 \\
\hline Sub- total 2: Costo mano de obra secado & 217,65 \\
\hline Costo total de Mano de Obra Directa (Stl + St2) & $1.283,8$ \\
\hline
\end{tabular}

Fuente: elaboración propia, a partir de los datos del estudio.

${ }^{*}$ Costos de Mano de Obra Directa: cosecha y secado.

El costo total de producción por hectárea asciende a US $\$ 10.055,69$, de ellos el $42 \%$ es variable y $58 \%$ es fijo. Finalmente, el costo unitario del kilo de ciruela deshidratada asciende a US $\$ 1,4$ el kilo, con un rendimiento de 7.200 kilos por hectárea, US\$1,26 el kilo, con un rendimiento de 8.000 kilos por hectárea, y US\$1,15 el kilo, con un rendimiento de 8.800 kilos por hectárea. Comparado con el estudio realizado por ODEPA, 2015 en la región de O’higgins, se refleja una desviación de 65\%, 59\% y 55\% del costo para los respectivos niveles de producción, sin considerar el efecto inflación el que asciende a 6,1\% entre abril de 2015 y diciembre de 2016, fechas de los respectivos estudios. Ver la tabla 8 .

TABLA 8

Estructura de costo unitario de producción del kilo de Ciruela Deshidratada

\begin{tabular}{|l|r|r|r|}
\hline \multicolumn{1}{|c|}{ Ciruelo Europeo } & \multicolumn{3}{c|}{ Vendimiento Kg/ ha } \\
\hline Rendimiento por hectárea (kilogramos/hectárea) & 7.200 & 8.000 & 8.800 \\
\hline Costo total depreciación Ciruelos: US\$ 1203,48 & 0,17 & 0,15 & 0,14 \\
\hline Costo total de Mano de Obra Directa: US \$1.283,8 & 0,18 & 0,16 & 0,15 \\
\hline Costo total de Costos Indirectos: US \$ 7.568,41 & 1,05 & 0,95 & 0,86 \\
\hline Costo total por kilogramo de ciruelas deshidratada: US\$ $\mathrm{kg}$. & 1,4 & 1,26 & 1,15 \\
\hline $\begin{array}{l}\text { Costo total por kilogramo de ciruelas deshidratada, según } \\
\text { ODEPA: US\$ } \mathrm{kg} \text {. }\end{array}$ & 0,85 & 0,79 & 0,74 \\
\hline
\end{tabular}

(Valor US\$ $=678,28$ )

Fuente: elaboración propia, a partir de los datos del estudio.

En términos metodológicos la diferencia radica en que el estudio de ODEPA, realizado el año 2015 sólo considera los costos de mano de obra, costos indirectos e insumos de un solo periodo de producción, y no 
considera el impacto de la depreciación de los árboles ciruelos (activos no corrientes) en el kilo de ciruela deshidratada, que representan un $20 \%$.

\section{Conclusión}

La fruticultura en Chile es intensiva en costos fijos. El costo total de la hectárea de Ciruelo D’Agen al finalizar el segundo año de crecimiento, es de a US\$19.255,64 y US\$38,51 por cada planta, con base en el método de Costeo Absorbente, es decir, información válida para efectos de tomar decisiones que impactan en el mediano y largo plazo. El costo total de producción por hectárea de ciruelos, al tercer año, asciende a US\$10.055,69, de ellos el $42 \%$ es variable y $58 \%$ es fijo. El costo unitario del kilo de ciruela deshidratada asciende a US\$1,4 el kilo, con un rendimiento de 7.200 kilos por hectárea, US\$ 01,26 el kilo, con un rendimiento de 8.000 kilos por hectárea, y US\$1,15 el kilo, con un rendimiento de 8.800 kilos por hectárea.

Es importante destacar, desde una perspectiva multidisciplinaria, la valoración de un activo no corriente, como lo es el Ciruelo, y su impacto, a través de su respectiva depreciación, en el costo de kilo de ciruela deshidratada. Al no considerar dicho impacto, el costo de producción está subvaluado, en tanto, los márgenes de venta, a partir de los cuales los productores toman decisiones, estarían sobrevalorados, para este caso en un $20 \%$.

De ahí la importancia de establecer una metodología, que establezca procedimientos sistemáticos para efectos de medir y evaluar los costos de producción en el sector frutícola, incorporando la dimensión técnica, económica y financiera. Lo anterior, teniendo presente que dicha información se necesita para tomar decisiones que impactan tanto en el corto, como en el mediano y largo plazo.

\section{Referencias}

Alvear, S., \& Rodríguez, P. (2006). Estimación del costo por kilómetro y de los márgenes de una empresa de transporte de carga, industria agrícola. Región del Maule, Chile. Revista Panorama Socioeconómico, 24(32), 48-57.

Anriquez, G. (2016). Desafíos en el mercado laboral para el desarrollo de la agricultura chilena. Consultado el 30 de agosto de 2018. Disponible en https://www.odepa.gob.cl/wp-content/uploads/2016/12/EstMercLaboralAgri cola.pdf

Argilés-Bosch, J. (2007). La información contable en el análisis y predicción de viabilidad de las explotaciones agrícolas. Revista de Economía Aplicada, 15(44), 109-135.

Argilés-Bosch, J., Miarons, M., Garcia-Blandon, J., Benavente, C., \& Ravenda, D. (2017). Usefulness of fair valuation of biological assets for cashflow prediction. Revista Española de Financiación y Contabilidad, 47(2), 157-180, h ttps://doi.org/10.1080/02102412.2017.1389549

Arimany, N., Farreras, M., \& Rabaseda, J. (2013). Alejados de la NIC 41. ¿Es correcta la valoración del patrimonio neto de las empresas agrarias? Economia Agraria y Recursos Naturales, 13(1), 27-50. https://doi.org/10.7201/ earn.2013.01.02

Cobianchi, D., Bergamini, A., \& Cortesi, A. (1989). El Ciruelo. Madrid: Mundi-Presa.

García, B., Gómez, J., \& Heras, E. (2018). Debt pressure and interactive use of control systems: Effects on cost of debt. Management Accounting Research, 40, 27-46. https://doi.org/10.1016/j.mar.2017.10.001

Gumucio, M. (2017). Análisis macrosectorial. ODEPA. Consultado el 9 de enero de 2017, en https://www.odepa.go b.cl/wp-content/uploads/2017/10/macroeconomia_octubre2017.pdf

Horngren, C., Foster, G., \& Datar, S. (2007). Contabilidad de costos: un enfoque gerencial. México DF: Prentice Hall S.A.

Jack, L., Florez-Lopez, R., \& Ramon-Jeronimo, J. (2018). Accounting, performance measurement and fairness in UK fresh produce supply networks. Accounting, Organizations and Society, 64, 17-30, http://doi.org/10.1016/j.ao s.2017.12.005 
Mallo, C., Kaplan, R., Meljem, S., \& Giménez, C. (2000). Contabilidad de costos y estratégica de gestión. Madrid: Prentice Hall Iberia.

Marvinney, E., Brodt, S., \& Kendall, A. (2016). Life Cycle Assessment: A tool for quantifying the environmental impacts of plum and prune production. Research Report. California Dried Plum Board. Consultado el $12 \mathrm{de}$ abril de 2017. Disponible en http://asi.ucdavis.edu/programs/sarep/research-initiatives/are/energy/lcaorchard

Normas Internacionales de Información Financiera, IFRS. (2017). Agricultura, NIC 41. Consultado el 18 de enero de 2017, en http://eifrs.ifrs.org

Normas Internacionales de Información Financiera, IFRS. (2017). Inventarios, NIC 2. Consultado el 18 de enero de 2017, en http://eifrs.ifrs.org.

Normas Internacionales de Información Financiera, IFRS. (2017). Marco Conceptual. Consultado el 18 de enero de 2017, en http://eifrs.ifrs.org

Normas Internacionales de Información Financiera, IFRS. (2017). Propiedades Plantas y Equipos, NIC 16. Consultado el 18 de enero de 2017 en http://eifrs.ifrs.org

Oficina de Estudios y Política Agrarias, ODEPA. (2015). Costo de producción ciruela para secado, región de O'Higgins. Consultado el 18 de enero de 2017 en https://www.odepa.gob.cl/wpcontent/uploads/2015/11/fic ha_costo_ciruela_secado_ohiggins_2015.pdf

Riveros, P., Aguirre, R., Ortega, J., \& Soto, S. (2016). La eficiencia técnica y una aproximación a sus determinantes: una medidapara mejorar la productividad en la fruticultura nacional. Oficina de Estudios y Política Agrarias, ODEPA. Consultado el 17 de enero de 2017 en http://www.odepa.cl/articulo

Tapia, B., \& González, C. (2012). Ciruelas secas: proyecciones de sobrestock y precios a la baja. Oficina de Estudios y Política Agrarias, ODEPA. Consultado el 17 de enero de 2017 en http://www.odepa.cl/articulo

\section{Notas}

* Artículo de investigación científica y tecnológica.

\section{Licencia Creative Commons CC BY 4.0}

Para citar este artículo: Alvear V., S., \& Figueroa S., K. (2018). Metodología de costos para los productos agrícolas, basada en las normas internacionales de contabilidad. Una aplicación en las ciruelas europeas variedad D’Agen. Cuadernos de Contabilidad, 19(48), 1-13. https://doi.org/10.11144/Javeriana.cc19-48. mcpa 